\title{
INTERAÇÃO TRITRÓFICA E INFLUÊNCIA DE PRODUTOS QUÍMICOS E VEGETAISS NO COMPLEXO: BRÁSSICAS X TRAÇA-DAS-CRUCÍFERAS X PARASITÓIDES DE OVOS
}

\author{
Tritrofic interaction and influence of insecticides and plant products on the complex: \\ brassica - diamondback moth - egg parasitoids
}

\author{
Robson Thomaz Thuler ${ }^{1}$, Sergio Antonio de Bortoli², Roberto Marchi Goulart ${ }^{3}$, \\ Cácia Leila Tigre Pereira Viana ${ }^{4}$, Dirceu Pratissoli ${ }^{5}$
}

\begin{abstract}
RESUMO
Objetivou-se avaliar a interação tritrófica no complexo hospedeiro-vegetal (brássicas) x praga/hospedeiro-natural Plutella xylostella Linnaeus x inseto-entomófago (parasitóides - Trichogrmma pretiosum Riley e T. exiguum Pinto \& Platner), associada a alguns produtos químicos e vegetais com efeito inseticida, utilizando-se os cultivares de repolho verde - Chato de quintal e híbrido Midori; roxo - Roxo precoce e Híbrido roxo - TPC00682; e couve manteiga - Geórgia e hibrido Geórgia HS20, pulverizadas com os inseticidas: lufenuron $(2,52 \mathrm{ml} / 100 \mathrm{~L})$ e deltametrina $(32 \mathrm{ml} / 100 \mathrm{~L})$, os produtos vegetais óleo de nim a $0,16 \%$ e extrato pirolenhoso a 3,0 \%, controle (água). Foi avaliada a interação das cultivares com os compostos por meio da exposição de lagartas recém-eclodidas aos produtos, avaliando-se os insetos nas fases de desenvolvimento até a emergência dos adultos. Para avaliar o efeito desses compostos sobre os parasitóides, foram empregados ovos de uma geração $\mathrm{F}_{2}$ de $P$. xylostella oriunda de lagartas alimentadas com folhas de brássicas, pulverizadas com esses produtos. A associação de produtos químicos ou vegetais, com efeito inseticida, com as cultivares de brássicas permitiu o manejo mais eficaz, especialmente na interação extrato pirolenhoso $\mathrm{x}$ a cultivar de repolho Chato de quintal. Observa-se que a interação entre as cultivares e os produtos pode ser prejudicial à atuação do parasitóide Trichogramma, sendo necessária uma avaliação criteriosa para minimizar o efeito sobre inimigos naturais.
\end{abstract}

Termos para indexação: Plutella xylostella, Trichogramma, crucíferas, controle biológico.

\section{ABSTRACT}

The aim of this work was to evaluate the tritrofic interaction in brassica complex: host-vegetable (brassica) vs. pest/ natural-host (Plutella xylostella) vs. entomophagous-insect (parasitoid - Trichogramma pretiosum and Trichogramma exiguum), combined with chemical and vegetable products with insecticide action. The cultivar used were: green cabbage - Chato de quintal and Midori hybrid; purple cabbage - Roxo Precoce and purple hybrid - TPC00682; kale - Georgia and Georgia hybrid HS20. They were sprayed using the insecticides - lufenuron $(2,52 \mathrm{ml} / 100 \mathrm{~L})$ and deltamethrin $(32 \mathrm{ml} / 100 \mathrm{~L})$; the vegetal products - neen oil $(0,16 \%)$ and pyroligneous extract $(3,0 \%)$; and water (check). The cultivars interaction was evaluated with the products, by larvae exposition to the treatments, being analyzed the different phases of development through adults emergence. To evaluate the effect of these products on the parasitoids, P. xylostella, eggs from F2 generation were used. Eggs of this pest were collected from adults fed with brassica leaves that were sprayed with the mentioned products. The insecticides association and vegetable products combined with brassica cultivars became more effective to manage the pest control, especially in the pyroligneous extract $x$ Chato de Quintal interaction. The interaction between the cultivars and products may be harmful to Trichogramma performance being necessary a discerning evaluation to minimize the effect on natural enemies.

Index terms: Plutella xylostella, Trichogramma, cruciferous, biological control.

(Recebido em 6 de junho de 2006 e aprovado em 8 de janeiro de 2008)

\footnotetext{
'Engenheiro Agrônomo, Doutor em Entomologia Agrícola, Departamento de Fitossanidade - Laboratório de Biologia e Criação de Insetos/LBCI Faculdade de Ciências Agrárias e Veterinárias/FCAV - Universidade Estadual Paulista/UNESP - Via de Acesso Professor Paulo Donato Castellane, Km 05 14884-900 - Jaboticabal, SP - rthuler@fcav.unesp.br

2Engenheiro Agrônomo, Doutor em Entomologia, Professor Titular - Departamento de Fitossanidade - Laboratório de Biologia e Criação de Insetos/LBCI Faculdade de Ciências Agrárias e Veterinárias/FCAV - Universidade Estadual Paulista/UNESP - Via de Acesso Professor Paulo Donato Castellane, Km 05 14884-900 - Jaboticabal, SP - bortoli@fcav.unesp.br

${ }^{3}$ Biólogo, Doutorando em Entomologia - Departamento de Fitossanidade - Laboratório de Biologia e Criação de Insetos/LBCI - Faculdade de Ciências Agrárias e Veterinárias/FCAV - Universidade Estadual Paulista/UNESP - Via de Acesso Professor Paulo Donato Castellane, Km 05 - $14884-900$ Jaboticabal, SP _ rm goulart@yahoo.com.br

${ }^{4}$ Engenheira Agrônoma, Doutoranda em Entomologia - Departamento de Fitossanidade - Laboratório de Biologia e Criação de Insetos/LBCI - Faculdade de Ciências Agrárias e Veterinárias/FCAV - Universidade Estadual Paulista/UNESP - Via de Acesso Professor Paulo Donato Castellane, Km 05 14884-900 - Jaboticabal, SP - caciat@yahoo.com.br

${ }^{5}$ Engenheiro Agrônomo, Doutor em Entomologia - Departamento de Produção Vegetal/DPV - Centro de Ciências Agrárias/CCA - Universidade Federal do Espírito Santo/UFES - Alto Universitário, s/n - Guararema - Cx. P. 16 - 29500-000 - Alegre, ES - dirceu@cca.ufes.br
} 


\section{INTRODUÇÃO}

As práticas culturais do sistema de produção de brássicas, por meio de plantios sucessivos e não eliminação dos restos culturais, principalmente para o repolho, juntamente com os fatores ambientais que favorecem o rápido crescimento das populações da traça-das-crucíferas Plutella xylostella (Linnaeus, 1758) (Lepidoptera: Plutellidae), aumentam o potencial de dano da praga, devido à possibilidade de sua multiplicação contínua (BARRANTES \& RODRIGUES, 1996; CASTELO BRANCO et al., 2003).

Para reduzir os prejuízos, a opção dos produtores de brássicas tem sido a aplicação intensiva de inseticidas; no entanto, tal prática não tem apresentado resultados satisfatórios ao longo dos anos, uma vez que aplicações, em até três vezes semanais, não reduziram os danos da traça, comprovando a existência de resistência dessas pragas a um grande número de inseticidas utilizados corriqueiramente (CASTELO BRANCO et al., 2001).

Além dessa prática de controle, outras são reconhecidamente comprovadas como eficientes no controle de $P$. xylostella, entre elas a utilização de cultivares resistentes que têm assumido papel relevante no manejo da praga (LIN et al., 1983, 1984; EIGENBRODE et al., 1990; ULMER et al., 2002); a utilização de plantas inseticidas na forma de extrato ou formulados por serem considerados produtos seletivos, com baixa toxicidade ao homem e eficiência contra várias espécies de insetos-praga (NEVES \& NOGUEIRA, 1996; SAXENA, 1989; SCHMUTTERER, 1987); e paralelamente, a alternativa de se utilizar insetos entomófagos, especificamente os parasitóides, torna-se mais real a cada dia, frente ao crescente número de laboratórios de produção que tem surgido e a utilização desses insetos, em várias regiões brasileiras (PARRA \& ZUCCHI, 2004).

Nos países com intenso cultivo de crucíferas já foram identificados mais de 90 espécies de parasitóides associados a $P$. xylostella, parasitando-a praticamente em todas as fases de desenvolvimento (TALEKAR \& SHELTON, 1993), sendo as principais espécies pertencentes aos gêneros Diadegma Cresson, Cotesia Cameron, Apanteles Foerster e Trichogramma Westwood, conforme relatado por Allam (1990), Cordero \& Cave (1992), Hirashima et al. (1989) e Yassem (1978).

Geralmente, os métodos utilizados para o controle da traça-das-crucíferas e para outros insetos-praga, são avaliados isoladamente ou apresentando-se o efeito do método sobre o inimigo natural. Como exemplo, pôde-se verificar em diversas pesquisas onde tem sido avaliado o efeito de inseticidas naturais: óleo de nim sobre Trichogramma minutum Riley, 1879; extratos de meliáceas sobre Trichogramma pretiosum Riley, 1879 e inseticidas químicos sobre T. pretiosum (CARVALHO et al., 2001, 2002, 2003; GONÇALVES-GERVÁSIO \& VENDRAMIM, 2004; LYONS et al., 2003; MOURA et al., 2004, 2005).

Apesar do conhecimento de várias técnicas de controle de pragas, pouco se sabe sobre a interação entre a resistência da planta $\mathrm{x}$ inseticidas $\mathrm{x}$ inseto-praga $\mathrm{x}$ insetoentomófago, e o impacto de um método de controle sobre outro, dificultando consideravelmente a possibilidade de implantação do manejo integrado de pragas (MIP) na cultura das brássicas.

Objetivou-se, neste trabalho avaliar a interação tritrófica no complexo das brássicas: hospedeiro-vegetal (brássicas) x praga/hospedeiro-natural $(P$. xylostella $) \mathrm{x}$ inseto-entomófago (parasitóides - T. pretiosum e Trichogramma exiguum Pinto \& Platner, 1978), aliada ao efeito de inseticidas químicos e produtos vegetais.

\section{MATERIAL E MÉTODOS}

O experimento foi conduzido no Laboratório de Biologia e Criação de Insetos (LBCI) da Faculdade de Ciências Agrárias e Veterinárias da Universidade Estadual Paulista (FCAV-UNESP) em Jaboticabal, sob temperatura de $25 \pm 1^{\circ} \mathrm{C}$, umidade relativa de $70 \pm 10 \%$ e fotofase de 14 horas.

Foi mantida para a experimentação uma criação de $P$. xylostella em laboratório, seguindo a metodologia desenvolvida por Barros \& Vendramim (1999) porém utilizando folhas de repolho e couve, pulverizadas com diferentes produtos. Dessa forma, folhas das cultivares de repolho (Brassica oleracea var. capitata) verde - Chato de quintal e híbrido Midori; roxo - Roxo precoce e Híbrido roxo; e para as cultivares de couve-manteiga (Brassica oleracea var. acephala) - Geórgia e Geórgia híbrido HS20 foram pulverizadas até o escorrimento, através de aparelho aerógrafo acoplado a um compressor de fluxo contínuo de ar, com os inseticidas químicos lufenuron 50CE, na dosagem de $2,52 \mathrm{ml} / 100 \mathrm{~L}$ de água, e deltametrina $25 \mathrm{CE}$, na dosagem de $32 \mathrm{ml} / 100 \mathrm{~L}$ de água; os produtos vegetais, com efeito inseticida, óleo de nim a $0,16 \%$, e extrato pirolenhoso a 3,0 $\%$. A água foi pulverizada como testemunha.

As concentrações letais médias (CL50), foram previamente obtidas para cada um, exceto a deltrametrina na qual utilizou-se a dosagem comercial recomendada, uma vez que para a população de $P$. xylostella, utilizada, a deltametrina não causou mortalidade.

Para se determinar a interação de efeitos entre as cultivares e os produtos, discos de folhas de $9 \mathrm{~cm}$ de diâmetro foram pulverizados, em cada face, com $0,5 \mathrm{ml}$ com soluções de cada produto, de forma idêntica ao realizado para a criação realizada em plantas tratadas com os inseticidas. Os discos foliares foram mantidos ao ar livre até a secagem e transferidos 
para placas de Petri, contendo papel filtro levemente umedecidos. Nas seções foliares colocaram-se 10 lagartas de P. xylostella recém-eclodidas, avaliando-se o seu desenvolvimento até a fase pupal. As pupas foram transferidas para células de placas de teste ELISA (Enzime Linked Immunosorbent Assay), até a emergência dos adultos; avaliou-se nessa etapa a viabilidade larval, pupal e razão sexual.

Após a obtenção da geração $\mathrm{F}_{2}$ de $P$. xylostella em laboratório, os ovos provenientes dessa geração foram expostos ao parasitismo de $T$. pretiosum e T. exiguum, utilizando insetos provenientes do LBCI/UNESP. Assim, para avaliação dos efeitos das cultivares e dos produtos, conjuntamente, sobre o terceiro nível trófico, para cada espécie do parasitóide, foram oferecidos 30 ovos de $P$. xylostella, provenientes das criações onde lagartas foram alimentadas com folhas tratadas com agrotóxicos na geração anterior. Os ovos foram colados com goma arábica a $35 \%$, em cartelas de cartolina azul celeste de 0,4 x 2,0 cm.

Para isso, foram coletados discos foliares das gaiolas de postura das diferentes criações que sofreram efeito dos agrotóxicos, e com o auxílio de um pincel levemente umedecido, os ovos foram destacados das folhas e aderidos às cartelas de cartolina.
Em cada tratamento, dez cartelas colocadas em tubos tipo Eppendorf ${ }^{\circledR}$ (capacidade para $2 \mathrm{ml}$ ), contendo uma fêmea de Trichogramma (ensaio realizado para as duas espécies de Trichogramma acima citadas), perfazendo um total de 30 tratamentos, em interação (6 cultivares x 4 inseticidas + água como testemunha).

O parasitismo foi permitido por $24 \mathrm{~h}$ e, posteriormente, as fêmeas foram retiradas dos tubos. Terminado o ciclo do parasitóide, com a emergência dos adultos, foram avaliadas as características biológicas: número de ovos parasitados, porcentagem de emergência e razão sexual. Os dados obtidos no experimento montado num delineamento inteiramente casualisado, foram submetidos à análise de variância (teste $\mathrm{F}$, nas probabilidades indicadas) e confrontadas pelo teste de Tukey $(\mathrm{P}=0,05)$.

\section{RESULTADOS E DISCUSSÃO}

A mortalidade larval de $P$. xylostella foi influenciada significativamente pelas cultivares, pelo inseticida químico, pelos produtos vegetais e pela ação conjunta desses fatores (Figura 1).

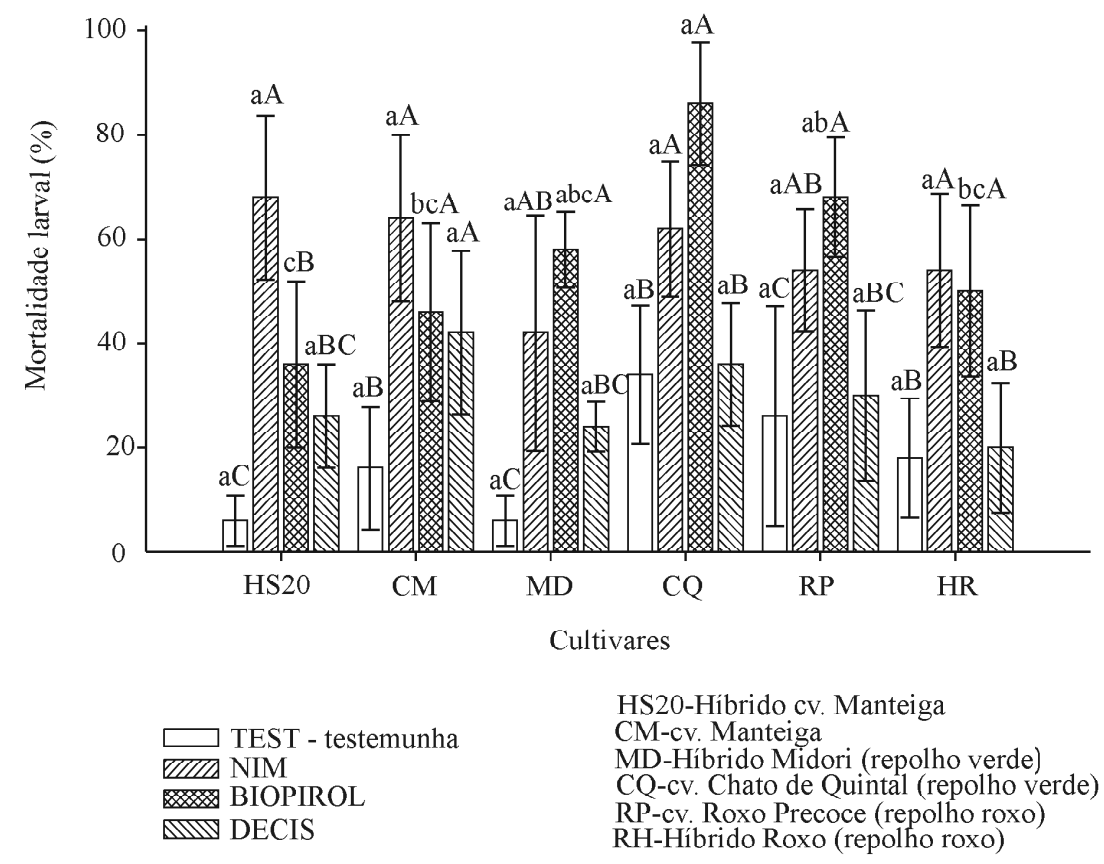

Teste $\mathrm{F}$ para cultivar $(\mathrm{P}=0,00029)$; $\mathrm{F}$ para inseticida $(\mathrm{P}=0,0001)$; F para interação $(\mathrm{P}=0,01449) \mathrm{C} . \mathrm{V} .(\%)=38,747$

* Médias seguidas pela mesma letra maiúscula para inseticida dentro de cultivar e, minúscula para cultivar dentro de inseticida, não diferem entre si; Tukey $(\mathrm{P}=0,05)$.

Figura 1 - Mortalidade larval $( \pm \mathrm{IC})$ de Plutella xylostella, alimentada com diferentes cultivares de brássicas contaminadas com alguns produtos químicos e vegetais com efeito inseticida. 
O efeito das cultivares sobre a mortalidade larval foi observado apenas dentro do tratamento com extrato pirolinhoso, sendo que a cultivar Chato de quintal teve uma ação interativa com o produto, elevando a mortalidade em relação aos demais tratamentos (Figura 1).

A não-verificação do efeito das cultivares possivelmente está relacionada ao tempo de observação e à fase analisada, uma vez que, Barros \& Vendramim (1999) constataram que os efeitos são mais evidentes quando se avalia um maior número de parâmetros. Esse efeito isolado das cultivares sobre o desenvolvimento de P. xylostella não foi objetivo dessa pesquisa.

De forma geral, os produtos vegetais com efeito inseticida utilizados foram sempre mais eficientes que a deltametrina, causando mortalidade de até $86 \%$ (extrato pirolinhoso/Chato de quintal) e até $68 \%$ (óleo de nim/cultivar manteiga- Geórgia HS20) (Figura 1). Os dados obtidos para o inseticida lufenuron não foram apresentados em função da mortalidade total ter atingido $100 \%$, em quase todas as interações testadas, inviabilizando a obtenção da segunda geração de $P$. xylotella para avaliação do efeito sobre o parasitóide.

Em relação à fase de pupa, não foi constatado efeito significativo para nenhum tratamento.

Os resultados observados para o efeito da interação das cultivares com os produtos químicos e vegetais, testados sobre as características biológicas da traça-dascrucíferas, demonstraram a eficiência de manejo, que pode não ser alcançada com a utilização dessas técnicas, em campos de plantio de brássicas.

Nas avaliações para o efeito da interação cultivares $x$ inseticidas $\mathrm{x}$ praga, sobre o terceiro nível trófico (Trichogramma), observou-se que os híbridos HS20, Midori e Híbrido roxo foram os que mais afetaram o número de ovos parasitados (Tabela 1), sendo o menor valor encontrado para o Híbrido Roxo, apesar desse não diferir significativamente dos demais híbridos (Tabela 2). Esse resultado demonstra que, em função da cultivar utilizada, pode haver incompatibilidade na interação resistência e parasitismo.

A interação entre as cultivares e os produtos químicos e vegetais utilizados, também influenciou negativamente o parasitismo por T. exiguum, sendo observado que, assim como para $P$. xylostella ( $2^{\circ}$ nível trófico), o tratamento que mais afetou o parasitóide ( $3^{\circ}$ nível) foi o extrato pirolinhoso/Roxo precoce, obtendo-se o menor número de ovos parasitados. Para os demais tratamentos, as interações deltametrina/Chato de quintal, deltametrina/Híbrido roxo, extrato pirolinhoso/ Híbrido roxo, extrato pirolinhoso/Chato de quintal, extrato de nim/Chato de quintal e extrato de nim/Híbrido roxo, foram aqueles onde o parasitismo foi o mais influenciado (Tabela 2).

Os efeitos dos híbridos sobre a porcentagem de parasitismo, no tratamento testemunha/cultivares, com exceção para o cultivar HS20, foram idênticos aos observados para o número de ovos parasitados. Esses também foram idênticos às interações entre produtos/cultivares, com destaque para a interação deltametrina/Chato de quintal, com uma viabilidade de apenas $15 \%$ de parasitismo (Tabela 2).

Os parâmetros biológicos avaliados para T. exiguum também foram feitos para $T$. pretiosum, no entanto, essa espécie não apresentou a mesma eficiência de parasitismo para ovos de $P$. xylostella, com resultados inferiores aos observados para T. exiguum (Tabela 3 ).

Para T. pretiosum não foi possível separar o efeito dos híbridos de forma tão evidente, uma vez que o número de ovos parasitados e a porcentagem de emergência, foram sempre menores evidenciando a não-preferência dessa espécie, por ovos de P. xylostella (Tabela 3 ).

Em relação à razão sexual dos insetos emergidos e em função da baixa porcentagem de parasitismo observada em alguns tratamentos, esses resultados não foram apresentados.

Tabela 1 - Número de ovos $( \pm \mathrm{IC})$ de Plutella xylostella, alimentada com diferentes cultivares de brássicas contaminadas com produtos químicos e vegetais com efeito inseticida e parasitados por Trichogramma exiguum.

\begin{tabular}{lccccc}
\hline \multirow{2}{*}{ Cultivares/híbridos } & \multicolumn{5}{c}{ Produtos químicos } \\
\cline { 2 - 6 } & $\mathrm{n}$ & Testemunha & nim & extrato pirolenhoso & deltametrina \\
\hline HS20 & 10 & $8,1 \pm 3,0 \mathrm{bA}$ & $14,5 \pm 5,2 \mathrm{aA}$ & $15,1 \pm 5,4 \mathrm{aA}$ & $7,9 \pm 5,3 \mathrm{abA}$ \\
Manteiga & 10 & $16,2 \pm 3,7 \mathrm{aA}$ & $10,5 \pm 1,8 \mathrm{abA}$ & $13,7 \pm 3,4 \mathrm{aA}$ & $12,9 \pm 3,5 \mathrm{aA}$ \\
Midori & 10 & $2,2 \pm 4,1 \mathrm{bB}$ & $8,4 \pm 2,4 \mathrm{abAB}$ & $13,7 \pm 3,3 \mathrm{aA}$ & $15,3 \pm 4,9 \mathrm{aA}$ \\
Chato de quintal & 10 & $17,7 \pm 2,0 \mathrm{aA}$ & $5,4 \pm 4,7 \mathrm{bB}$ & $3,0 \pm 3,0 \mathrm{bB}$ & $2,0 \pm 3,5 \mathrm{bB}$ \\
Roxo precoce & 10 & $18,4 \pm 6,2 \mathrm{aA}$ & $12,8 \pm 5,5 \mathrm{abA}$ & $1,2 \pm 0,9 \mathrm{bB}$ & $15,7 \pm 3,7 \mathrm{aA}$ \\
Híbrido roxo & 10 & $1,7 \pm 1,8 \mathrm{bA}$ & $5,9 \pm 4,7 \mathrm{bA}$ & $2,5 \pm 3,2 \mathrm{bA}$ & $2,5 \pm 2,3 \mathrm{bA}$ \\
\hline C.V.(\%) & \multicolumn{7}{c}{66,8} &
\end{tabular}

F para cultivar $(\mathrm{P}=0,00001)$; F para inseticida $(\mathrm{P}=\mathrm{ns})$; F para interação $(\mathrm{P}=0,00001)$.

* Médias seguidas pela mesma letra minúscula na coluna e maiúscula na linha, não diferem entre si; Tukey $(\mathrm{P}=0,05)$ 
Tabela 2 - Porcentagem de emergência $( \pm \mathrm{IC})$ de Trichogramma exiguum em ovos de Plutella xylostella, alimentada com diferentes cultivares de brássicas contaminadas com produtos químicos e vegetais com efeito inseticida.

\begin{tabular}{lccccc}
\hline \multirow{2}{*}{ Cultivares/híbridos } & \multicolumn{5}{c}{ Inseticidas químicos } \\
\cline { 2 - 6 } & $\mathrm{n}$ & Testemunha & nim & extrato pirolinhoso & deltametrina \\
\hline HS20 & 10 & $98,7 \pm 1,7 \mathrm{aA}$ & $99,6 \pm 0,7 \mathrm{aA}$ & $97,3 \pm 2,5 \mathrm{aA}$ & $100,0 \pm 0,0 \mathrm{aA}$ \\
Manteiga & 10 & $100,0 \pm 0,0 \mathrm{aA}$ & $100,0 \pm 0,0 \mathrm{aA}$ & $89,9 \pm 5,9 \mathrm{aA}$ & $98,3 \pm 2,2 \mathrm{aA}$ \\
Midori & 10 & $20,0 \pm 26,1 \mathrm{bB}$ & $100,0 \pm 0,0 \mathrm{aA}$ & $100,0 \pm 0,0 \mathrm{aA}$ & $100,0 \pm 0,0 \mathrm{aA}$ \\
Chato de quintal & 10 & $93,6 \pm 7,0 \mathrm{aA}$ & $48,6 \pm 31,9 \mathrm{bB}$ & $38,4 \pm 30,9 \mathrm{bBC}$ & $15,0 \pm 20,9 \mathrm{bC}$ \\
Roxo precoce & 10 & $100,0 \pm 0,0 \mathrm{aA}$ & $100,0 \pm 0,0 \mathrm{aA}$ & $50,0 \pm 32,7 \mathrm{bB}$ & $100,0 \pm 0,0 \mathrm{aA}$ \\
Híbrido roxo & 10 & $30,0 \pm 29,9 \mathrm{bA}$ & $45,9 \pm 30,2 \mathrm{bA}$ & $26,8 \pm 27,1 \mathrm{bA}$ & $50,0 \pm 32,7 \mathrm{bA}$ \\
\hline C.V.(\%) & \multicolumn{7}{c}{}
\end{tabular}

F para cultivar ( $\mathrm{P}=0,00001)$; F para inseticida $(\mathrm{P}=0,03580)$; F para interação $(\mathrm{P}=0,00001)$.

* Médias seguidas pela mesma letra minúscula na coluna e maiúscula na linha, não diferem entre si; Tukey $(\mathrm{P}=0,05)$

Tabela 3 - Número de ovos parasitados e porcentagem de emergência ( \pm IC) de Trichogramma exiguum e T. pretiosum, provenientes do parasitismo de ovos de Plutella xylostella, alimentada com diferentes cultivares de brássicas contaminadas com produtos químicos e vegetais com ação inseticida.

\begin{tabular}{lccccc}
\hline \multirow{2}{*}{ Cultivares/híbridos } & \multicolumn{3}{c}{ Número de ovos parasitados } & \multicolumn{2}{c}{ Porcentagem de emergência } \\
\cline { 2 - 6 } & $\mathrm{n}$ & T. exiguum & T. pretiosum & T. exiguum & T. pretiosum \\
\hline HS20 & 10 & $8,1 \pm 3,0 \mathrm{bA}$ & $3,6 \pm 4,0 \mathrm{aB}$ & $98,7 \pm 1,7 \mathrm{aA}$ & $60,0 \pm 32,0 \mathrm{bA}$ \\
Manteiga & 10 & $16,2 \pm 3,7 \mathrm{aA}$ & $0,3 \pm 0,6 \mathrm{aB}$ & $100,0 \pm 0,0 \mathrm{aA}$ & $10,0 \pm 19,6 \mathrm{bcB}$ \\
Midori & 10 & $2,2 \pm 4,1 \mathrm{bcA}$ & $2,2 \pm 2,9 \mathrm{aB}$ & $20,0 \pm 26,1 \mathrm{bA}$ & $50,0 \pm 32,6 \mathrm{abA}$ \\
Chato de quintal & 10 & $17,7 \pm 2,0 \mathrm{aA}$ & $1,0 \pm 1,1 \mathrm{aB}$ & $93,6 \pm 7,0 \mathrm{aA}$ & $30,0 \pm 29,9 \mathrm{abcB}$ \\
Roxo precoce & 10 & $18,4 \pm 6,2 \mathrm{aA}$ & $1,6 \pm 1,7 \mathrm{aB}$ & $100,0 \pm 0,0 \mathrm{aA}$ & $40,0 \pm 32,0 \mathrm{abcB}$ \\
Híbrido roxo & 10 & $1,7 \pm 1,8 \mathrm{cA}$ & $0,0 \pm 0,0 \mathrm{aA}$ & $30,0 \pm 29,9 \mathrm{bA}$ & $0,0 \pm 0,0 \mathrm{cA}$ \\
\hline C.V.(\%) & \multicolumn{7}{c}{} \\
\hline
\end{tabular}

F para cultivar ( $\mathrm{P}=0,00001)$; $F$ para inseticida $(\mathrm{P}=0,03580)$; F para interação $(\mathrm{P}=0,00001)$.

* Médias seguidas pela mesma letra minúscula na coluna e maiúscula na linha, não diferem entre si; Tukey $(\mathrm{P}=0,05)$

\section{CONCLUSÕES}

- A associação de produtos químicos e vegetais com efeito inseticida sobre as cultivares de brássicas influenciou a mortalidade de lagartas de Plutella xylostella, o número de ovos parasitados e a porcentagem de emergência dos parasitóides.

- A associação de resistência de plantas x controle de pragas com produtos químicos ou vegetais $\mathrm{x}$ controle biológico, num programa de manejo integrado em brássicas, necessita ser avaliada com profundidade, para se minimizar o efeito sobre parasitóide de ovos do gênero Trichogramma.

\section{AGRADECIMENTOS}

À FAPESP (Fundação de Amparo à Pesquisa do Estado de São Paulo), pela concessão da bolsa de estudos ao primeiro autor e pela reserva técnica disponibilizada para o desenvolvimento desta pesquisa.

\section{REFERÊNCIAS BIBLIOGRÁFICAS}

ALLAM, N. M. Diamondback moth and its natural enemies in Jamaica and some other Caribbean Islands. In: INTERNATIONAL WORKSHOP OF DIAMONDBACK MOTH AND OTHER CRUCIFERS PESTS, 2., 1990, Taiwan:

Proceedings... Taiwan: Asian Vegetable Research an Development Center, 1990. p. 233-243.

BARRANTES, A. J. A.; RODRIGUEZ, V. C. L. Abundancia estacional y dano de Plutella xylostella (L.) (Lepidoptera: Plutellidae) y el cultivo de repollo, durante la epoca seca en Alfaro Ruiz, Alajuela, Costa Rica. Manejo Integrado de Plagas, Chillan, v. 39, p. 17-24, 1996. 
BARROS, R.; VENDRAMIM, J. D. Efeito de cultivares de repolho, utilizadas para criação de Plutella xylostella (L.) (Lep.: Plutellidae) no desenvolvimento de Trichogramma pretiosum (Riley) (Hym.: Trichogrammatidae). Anais da Sociedade Entomológica do Brasil, Londrina, v. 28, n. 3, p. 469-476, 1999.

CARVAlHO, G. A.; PARRA, J. R. P.; BAPTISTA, J. C. Seletividade de alguns produtos fitossanitários a duas linhagens de Trichogramma pretiosum Riley, 1879 (Hymenoptera: Trichogrammatidae). Ciência e Agrotecnologia, Lavras, v. 25, n. 3, p. 583-591, 2001.

CARVALHO, G. A.; PARRA, J. R. P.; BAPTISTA, J. C. Efeito de produtos fitossanitários utilizados na cultura do tomateiro (Lycopersicon esculentum Mill.) sobre Trichogramma pretiosum Riley, 1879 nas gerações f1 e f2 em ovos de Anagasta kuehniella (Zeller, 1879). Ciência e Agrotecnologia, Lavras, v. 27, n. 2, p. 295-304, 2003.

CARVALHO, G. A.; REIS, P. R.; MORAES, J. C.; FUINI, L. C.; ROCHA, L. C. D.; GOUSSAIN, M. M. Efeitos de alguns inseticidas utilizados na cultura do tomateiro (Lycopersicon esculentum Mill.) a Trichogramma pretiosum Riley, 1879 (Hymenoptera: Trichogrammatidae). Ciência e Agrotecnologia, Lavras, v. 26, n. 6, p. 1160-1166, 2002.

CASTELO BRANCO, M.; FRANÇA, F. H.; MEDEIROS, M. A.; LEAL, J. G. T. Uso de inseticidas para o controle da traça-do-tomateiro e traça-das-crucíferas: um estudo de caso. Horticultura Brasileira, Brasília, v. 19, n. 1, p. 60-63, 2001.

CASTELO BRANCO, M.; FRANÇA, F. H.; PONTES, L. A.; AMARAL, P. S. T. Avaliação da suscetibilidade a inseticidas em populações de traça-das-crucíferas de algumas áreas do Brasil. Horticultura Brasileira, Brasília, v. 21, n. 3, p. 549-552, 2003.

CORDERO, J.; CAVE, R. D. Natural enemies of Plutella xylostella (L.) (Lepidoptera: Plutellidae) on crucifers in Honduras. Entomophaga, Paris, v. 37, n. 3, p. 397-407, 1992.

EIGENBRODE, S. D.; SHELTON, A. M.; DICKSON, H. Two types of resistance to the diamondback moth (Lepidoptera:
Plutellidae) in cabbage. Environmental Entomology, College Park, v. 19, n. 4, p. 1086-1090, 1990.

GONÇALVES-GERVÁSIO, R. C. R.; VENDRAMIM, J. D. Efeito de extratos de meliáceas sobre o parasitóide de ovos Trichogramma pretiosum Riley (Hymenoptera: Trichogrammatidae). Neotropical Entomology, Londrina, v. 33, n. 5, p. 607-612, 2004.

HIRASHIMA, Y.; ABE, M.; TADAUCHI, O.; KONISHI, K.; MAETO, K. The hymenopterous parasitoids of the diamondback moth Plutella xylostella (Lepidoptera: Yponomeutidae) in Japan. Esakia, Fukuoka, v. 28, p. 63-74, 1989.

LIN, J.; DICKSON, M. H.; ECKENRODE, C. J. Resistance of Brassica lines to the diamondback moth (Lepidoptera: Yponomeutidae) in the field, and inheritance of resistance. Journal of Economic Entomology, Lanham, v. 77, p. 12931296, 1984

LIN, J.; ECKENRODE, C. J.; DICKSON, M. H. Variation in Brassica oleracea resistance to diamondback moth (Lepidoptera: Plutellidae). Journal of Economic Entomology, Lanham, v. 76, p. 1423-1427, 1983.

LYONS, D. B.; HELSON, B. V.; BOURCHIER, R. S.; JONES, G. C.; MCFARLANE, J. W. Effects of azadirachtin-based insecticides on the egg parasitoid Trichogramma minutum (Hymenoptera: Trichogrammatidae). The Canadian Entomologist, Ottawa, v. 135, n. 5, p. 685-695, 2003.

MOURA, A. P.; CARVALHO, G. A.; RIGITANO, R. L. O. Efeito residual de novos inseticidas utilizados na cultura do tomateiro sobre Trichogramma pretiosum Riley, 1879 (Hymenoptera: Trichogrammatidae). Acta Scientiarum Agronomy, Maringá, v. 26, n. 2, p. 231-237, 2004.

MOURA, A. P.; CARVALHO, G. A.; RIGITANO, R. L. O. Toxicidade de inseticidas utilizados na cultura do tomateiro a Trichogramma pretiosum. Pesquisa Agropecuária Brasileira, Brasília, v. 40, n. 3, p. 203-210, 2005.

NEVES, B. P.; NOGUEIRA, J. C. M. Cultivo e utilização do nim indiano (Azadirachta indica A. Juss.). Brasília, DF: Embrapa-CNPAF-APA, 1996. 32 p. (Circular técnica, 28). 
PARRA, J. R. P.; ZUCCHI, R. A. Trichogramma no Brasil: viabilidade de uso após vinte anos de pesquisa. Neotropical Entomology, Londrina, v. 33, n. 3, p. 271-282, 2004.

SAXENA, R. C. Inseticides from Neem. In: ARNASON, J. T.; PHILOGENE, B. J. R.; MORAND, P. (Eds.). Insecticides of plant origin. Washington, DC: American Chemical Society, 1989. p. 110-129.

SCHMUTTERER, H. Insect growth-disrupting and fecundity-reducing ingredients from the neem and chinaberry trees. In: MORGAN, E. D.; MANDAVA, N. B. (Eds.). CRC handbook of natural pesticides: insect growth regulators: part B. Washington: CRC, 1987. v. 3, p. 119-167.
TALEKAR, N. S.; SHELTON, A. M. Biology, ecology and management of the diamondback moth. Annual Review of Entomology, Palo Alto, v. 38, p. 275-301, 1993.

ULMER, B. C.; GILLOTT, C.; WOODS, D.; ERLANDSON, M. Diamondback moth, Plutella xylostella (L.), feeding and oviposition preferences on glossy and waxy Brassica rapa (L.) lines. Crop Protection, Guildford, v. 21, n. 4, p. 327-331, 2002.

YASSEN, M. The establishment of two parasites of the diamondback moth Plutella xylostella (Lepidoptera: Plutellidae) in Trinidad, W. I. Entomophaga, Paris, v. 23, n. 2, p. 111-114, 1978. 